\title{
THE OPTICAL PROPERTIES OF InGaAs(P)/InP UNDER GROUP V SUBLATTICE TWO-PHASE INTERDIFFUSION
}

\author{
E. Herbert Li*, Joseph Micallef ${ }^{* *}$, and W.C. Shui"** \\ *University of Hong Kong, Dept. of EEE, Pokfulam Road, Hong Kong, ehli@hkueee.hku.hk \\ **University of Malta, Department of Microelectronics, Malta \\ ***Hong Kong Baptist University, Department of Mathematics, Hong Kong.
}

\section{Abstract}

Using the fundamental transition state, we will investigate the two phase interdiffusion of group V sublattice in a strained lattice matched InGaAs/InP quantum well (QW) structures. The model employs three parameters namely the diffusion coefficients in the barrier $\left(D_{b}\right)$ and in the well $\left(D_{w}\right)$ and the concentration ratio $(k)$ of the diffused species at the heterstructure. The QW model includes the effects of strain and the exciton. A pseudo time dependent calculation is also considered and results are fitted to the reported experimental data. These parameters which characterize the diffusion mechanism can be measure to form a better understanding of the interdiffsion process for group $\mathrm{V}$ sublattice.

\section{Introduction}

Lattice-matched InGaAs/InP QW structures are of considerable interest in photonic applications such as waveguides [1] and modulators [2,3] since they enable device operation in the $1.3 \mu \mathrm{m}$ to $1.55 \mu \mathrm{m}$ wavelength range, they are important for optical communication systems. Diffused QW (DFQW) consisting of InGaAs/GaAs and $\mathrm{AlGaAs} / \mathrm{GaAs}$ material systems have been studied in detail. The mechanism of group III only [4] and the inclusive group III and V $[4,5]$ interdiffusion has been heavily studied and the mechanism is very well understood whereas for group $\mathrm{V}$ interdiffusion[5], it is still subject to active investigation. The group V interdiffusion poses interesting aspects both in the mechanism of the diffusion process and the potential for band-gap engineering. The as-grown square weil compositional profile is modified to a graded profile thereby altering the confinement profile and subband structure in the QW. The recent report [6a] on the group V sublattice diffusion was not satisfactory in the way that the data was ill-fitted. The acquired fitting data did rot fully reflect the situation of the diffusion mechanism. For this purpose we need to gain further insight into the situation, by using our proposed model to mediate. The disordering process can thus provide a useful tool for tayloring device properties. InGaAs/InP DFQW structures are more complicated whereby interdiffusion can occur for both group III (In, Ga) and group V (As, P) species. Detailed knowledge of the physical processes in InGaAs/InP QW is very important for a more advantageous design and application of related devices.

\section{Modeling}

A number of models have been presented for investigating diffusion involving the use of Error functions in approximating the solution. Assumptions such as having semi-infinite substrates length, where the diffusing species have negligible effects on reflections from boundaries are not true. In real cases, the profiles have symmetry around a boundary. It may be well suited for recognized smooth diffused concentration profile systems where there are no 
discontinuities at boundaries. $\mathrm{In}_{0.53} \mathrm{Ga}_{0.47} \mathrm{As} / \mathrm{InP}$ DFQW are more complicated than those of other material system since interdiffusion can occur for both group III (In, Ga) and group V (As, P) atoms. It is known that the group $\mathrm{V}$ diffusion will result in a tensile strained well and has been suggested [7] that As composition in the well layer decreases evenly and that the As atoms accumulate near the heterostructure interface. Fujii[8] proposed a model of interdiffusion that has large compositional discontinuity of group $\mathrm{V}$ atom at the interface even after interdiffusion has taken place. The as-grown lattice-matched $\operatorname{In}_{x} \mathrm{Ga}_{1-x} \mathrm{As}_{y} \mathrm{P}_{1-\mathrm{y}} / \mathrm{InP}$ material with $\mathrm{y} \approx 2.2(1-\mathrm{x})$ [9] exhibit no strain phenomena. The system to be modeled consists of an $\operatorname{In}_{0.53} \mathrm{Ga}_{0.47} \mathrm{As}$ layer sandwiched between thick InP barriers. The assumption made on this system in comparison to that of other material system is that there is a concentration boundary discontinuity which prevails at all times. In order to give a comprehensive DFQW model of group $\mathrm{V}$, two phase constituents were used in the three body system. A numerical method using the finite difference method was used to give the model the flexibility of setting up different boundaries and initial conditions. Also, to do away with the constraints of required parameters in the interdiffusion process, we used this numerical method to comply to the restrictions in order to achieve an analytical solution. For a heterostructure of QW structure, the interdiffusion process can be represented by linear diffusion equations. For a structure of a middle layer (InGaAs) surrounded by another outside layers (InP), the diffusion equations for describing this system are:

$$
\begin{array}{ll}
\frac{\partial C_{b}(z, t)}{\partial t}=D_{b} \frac{\partial^{2} C_{b}(z, t)}{\partial z^{2}} & \text { for } t \geq 0, z \geq L \\
\frac{\partial C_{w}(z, t)}{\partial t}=D_{w} \frac{\partial^{2} C_{w}(z, t)}{\partial z^{2}} & \text { for } t \geq 0,|z|<L \\
\frac{\partial C_{b}^{\prime}(z, t)}{\partial t}=D_{b} \frac{\partial^{2} C_{b}^{\prime}(z, t)}{\partial z^{2}} & \text { for } t \geq 0, z \leq-L
\end{array}
$$

with the boundary condition: $C_{b}(z, t)=k C_{w}(z, t)$; for $z \rightarrow L$

$$
\begin{aligned}
& C_{b}^{\prime}(z, t)=k C_{w}(z, t) ; \text { for } z \rightarrow-L \\
& D_{b} \frac{\partial C_{b}(z, t)}{\partial z}=D_{w} \frac{\partial C_{w}(z, t)}{\partial z} ; \text { for } z \rightarrow L \\
& D_{b} \frac{\partial C_{b}^{\prime}(z, t)}{\partial z}=D_{w} \frac{\partial C_{w}(z, t)}{\partial z} ; \text { for } z \rightarrow-L
\end{aligned}
$$

where $\pm \mathrm{L}$ is distance of interface from the well centre; $C_{b}, C_{b}$ are concentration of diffusion species in the outside layers; $C_{w}$ is concentration in the well layer; $D_{b}$ is diffusion coefficients in the outside layers; $D_{w}$ is diffusion coefficients in the well layer; $k$ is the interfacial distribution ratio of concentration. By dividing the model grid into finite time and position step, and by using the initial profile as the initial condition in a positional array, the partial differential equations is solved. The solution to the finite difference methods give rise to the concentrations of the diffused species as a profile across the system, $C(z)$, normalized over the system.

If the well is within the critical thickness regime so that no dislocation occur in the relaxing of the homogeneous stress effect, the strain effect will vary continuously across the $\mathrm{QW}$. Subsequently the carrier effective mass $\mathrm{m}_{\mathrm{r}}{ }^{*}$ will have a spatial dependence on concentration and it has a form $\mathrm{m}_{\mathrm{r}}^{*}(\mathrm{z})=\mathrm{m}_{\mathrm{r}}^{*}(\tilde{x}, \tilde{y})$, where $\mathrm{m}_{\mathrm{r}}{ }^{*}(\mathrm{x}, \mathrm{y})$ is the bulk effective mass and $\mathrm{r}$ represents the 
electron (C) or heavy hole $(\mathrm{HH})$. These value are given in table (1). The strained bulk bandgap in the well, $\mathrm{E}_{\mathrm{g}}$ is also a function of the compositional profile so that the strained potential profile after interdiffusion is $\Delta \mathrm{E}_{\mathrm{r}}(\mathrm{x}, \mathrm{y})=\mathrm{Q}_{\mathrm{r}} \Delta \mathrm{E}_{\mathrm{g}}(\mathrm{x}, \mathrm{y})$, where $\mathrm{Q}_{\mathrm{r}}$ is the band offset ratio and $\Delta \mathrm{E}_{\mathrm{g}}$ is the unstrained bandgap offset. The DFQW will be coherently strained [23], with a biaxial hydrostatic strain parallel to the interfacial plane and a uniaxial shear strain perpendicular to the interfacial plane. A compressive (tensile) hydrostatic strain causes an increase (decrease) in the bandgap energy. The shear strain disrupts the cubic symmetry of the semiconductor and lifts the degeneracy of the heavy hole $(\mathrm{HH})$ band edges at the Brillouin zone centre $\Gamma$. The heavy hole band shifts towards (away from) the conduction band away from (towards) the conduction band [6]. In addition, the presence of strain induces coupling between the LH band to the spin-orbit split-off band [12]. The DFQW confinement potential, is therefore given by $U_{r}(z)=\Delta E_{r}(\widetilde{x}, \widetilde{y})$ $S_{\perp r}(\widetilde{x}, \widetilde{y}) \pm S_{\| r}(\widetilde{x}, \widetilde{y})$, where $S_{\perp r}(\widetilde{x}, \widetilde{y})=Q_{r} S_{\perp}(\widetilde{x}, \widetilde{y})$, the +ve sign represents the confined HH profile while the -ve sign represents the confined LH profile. Eq. (1) represents the carrier confinement profile for the general case, where interdiffusion on both sublattices takes place, but at different rates. For only group $V$ sublattice interdiffusion :

$$
\mathrm{U}_{\mathrm{r}}(\mathrm{z})=\Delta \mathrm{E}_{\mathrm{r}}(0.53, \widetilde{\mathrm{y}})-\mathrm{S}_{\perp \mathrm{r}}(0.53, \widetilde{\mathrm{y}}) \pm \mathrm{S}_{\| \mathrm{r}}(0.53, \widetilde{\mathrm{y}})
$$

The electron and hole subband structure at $\Gamma$ can be determined by considering the appropriate schrödinger equation from the BenDaniel-Duke model [39], using the envelope function scheme 40 with an effective mass approximation. The one-electron Schrodinger equation for the DFQW can be expressed as :

$$
-\frac{\hbar^{2}}{2} \frac{\mathrm{d}}{\mathrm{dz}}\left[\frac{1}{\mathrm{~m}^{*}{ }_{\mathrm{r}}(\mathrm{z})} \frac{\mathrm{d} \chi_{\mathrm{rl}}(\mathrm{z})}{\mathrm{dz}}\right]+\mathrm{U}_{\mathrm{r}}(\mathrm{z}) \chi_{\mathrm{rl}}(\mathrm{z})=\mathrm{E}_{\mathrm{rl}} \chi_{\mathrm{rl}}(\mathrm{z})
$$

where the growth direction $z$ is the confinement axis, $\chi_{\mathrm{rl}}(\mathrm{z})$ is the envelope waverunction, $\mathrm{E}_{\mathrm{rl}}$ is the quantized energy level with the subband energy at the bottom of the QW, and $l=p$ or q refers to the quantized subband energy levels for the electron and holes, respectively. This equation is solved numerical to obtain the eigen energy values $\left(E_{\mathrm{cp}}, \mathrm{E}_{\mathrm{vq}}\right)$, and the envelope wavefunction $\left(\chi_{c p}, \chi_{v q}\right)$. Hence the interband transitions energy can be determined. The electron-heavy hole transition energy, $E_{\mathrm{CHH} 11}$ can then be obtained from $E_{\mathrm{CHH} 11}=E_{\mathrm{gHH}}(\widetilde{\mathrm{x}}, \widetilde{\mathrm{y}})+\mathrm{E}_{\mathrm{c} 1}+\mathrm{E}_{\mathrm{HH} 1}$ evaluated at $\Gamma$, the lowest direct bandgap after disordering.

\section{Results and Discussion}

The InGaAs/InP DFQW ( $\left.\mathrm{L}_{d}=0\right)$ model for the as grown square $\mathrm{QW}$, has been validated with very good results, see Table 2 . The reported analysis of the experimental data [15] aroused skepticism in that large deviations were noticed from the experimental data. The longer annealing time period suggested perhaps one set of perameters, $D_{b}, D_{w}$, and $k$ did not fully reflect 


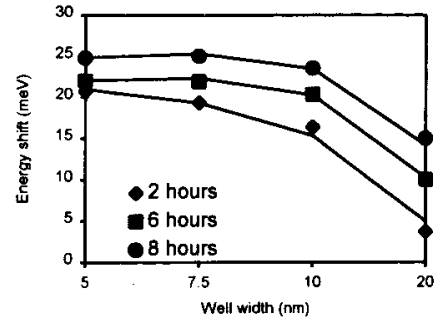

Fig. 1 Quantum energy shift as a function of well width for $\mathrm{In}_{0.53} \mathrm{Ga}_{0.47} \mathrm{As} / \mathrm{InP}$ quantum well after annealing at $650^{\circ} \mathrm{C}$ for 2, 6, $8 \mathrm{hrs}$

the mechanism of the diffusion and therefore might be time dependent on the parameters. By using our model to refit the data (using constants $D_{b}, D_{w}$ and $k$ ) only one set of parameters would favourably fit the same annealing time, thereby supporting our original idea that more than one set of parameters were needed to reflect the situation fully. Consequently it led to the belief that there exist different sets of parameter for different annealing times. This is contrary to previous reports [15], where the parameters are presumed to be constant throughout. Intuitively $D_{b}, D_{w}$ and $k$ is expected to exhibit some kind of time dependency because the spatial concentration of the diffused As varies across the QW which should have some real effects on the parameters. Alternatively, in order to show that the parameters show some form of time dependency, the experimental data were fitted using different set of parameters for different annealing times. In order to simulate a more realistic model, the fitting was divided into three different time intervals as the experimental data permitted. This used the concentration of diffused species across the DFQW after a time period to serve as the initial condition to the next time step, the process was then repeated for the subsequent time step. By doing so, a new set of parameters were determined for each step which was valid for that time span. Finally, three different set of parameters were determined and the result is shown in Table 3.

Due to the lack of experimental data available, the computed values of the time dependent parameters could not be substantiated further. But nonetheless it showed that parameters are time dependent, they do not remain constant. This would be more acceptable in real physical conditions. From Fig. 2, the value of $D_{b}, D_{w}$ and $k$ is plotted against time.
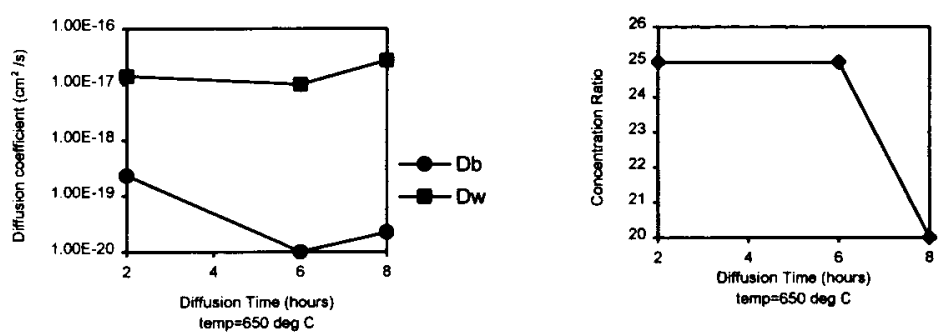

Fig. 2 (a) Stepwise interdiffusion coefficients as a function of time

(b) Stepwise concentration ratio at the heterostructure as a function of time

If the model does show the correct dependency then the reason for the trend shown in Fig. 2 can be explained that in reality the relationship beyond the interface is not only controlled 
by the simple ratio of concentration of the two sides, $C_{b}=k_{w}$ alone. Once the diffusion has been going on for a period of time, the material will pile up at the interface causing a drop in $D_{b}$, $D_{w}$ which further slow down the interdiffusion rate, but after a while the material which gathers at the interface clears out and the species go on unimpeded in the interdiffusion.

\section{Conclusion}

To describe interdiffusion of QW and to analyze the dependence of Quantum energy shift on annealing time, temperature and well width in lattice-matched $\mathrm{InGaAs} / \mathrm{InP}$ structure, we used a DFQW model. The formula correctly models the dependence and casts some light on the interdiffusion of the group $\mathrm{V}$ species.

Table 1 Material parameters of GaAs, InAs, $\mathrm{GaP}$ and $\mathrm{InP}$ at low temperature $(\leq 10 \mathrm{~K})$ used in the numerical calculations. $a_{0}$ is the lattice constant, $m_{0}$ is the electron mass in free space.

\begin{tabular}{|l|l|l|}
\hline & Unit & $\mathrm{In}_{0.53} \mathrm{Ga}_{0.47} \mathrm{As}_{\mathrm{y}} \mathrm{P}_{1-\mathrm{y}}$ \\
\hline $\mathrm{E}_{\mathrm{g}}$ & $\mathrm{eV}$ & $1.9143-1.0782 \mathrm{y}-0.0239 \mathrm{y}^{2}$ \\
\hline$\Delta_{\mathrm{o}}$ & $\mathrm{eV}$ & $0.09578-0.26589 \mathrm{y}$ \\
\hline $\mathrm{m}_{\mathrm{e}}$ & $\mathrm{m}_{\mathrm{o}}$ & $0.05039-0.006945 \mathrm{y}$ \\
\hline $\mathrm{m}_{\mathrm{hh}}$ & $\mathrm{m}_{\mathrm{o}}$ & $0.5534-0.212711 \mathrm{y}$ \\
\hline $\mathrm{c}_{11}$ & $\times 10^{11} \mathrm{dyn} / \mathrm{cm}^{2}$ & $12.1799-2.0169 \mathrm{y}$ \\
\hline $\mathrm{c}_{12}$ & $\times 10^{11} \mathrm{dyn} / \mathrm{cm}^{2}$ & $6.1172-1.0326 \mathrm{y}$ \\
\hline $\mathrm{a}_{\mathrm{o}}$ & $\AA$ & $5.672528+0.195099 \mathrm{y}$ \\
\hline $\mathrm{dE}_{\mathrm{g}} / \mathrm{dP}$ & $\mathrm{eV} / \mathrm{bar}$ & $9.481+1.236 \mathrm{y}$ \\
\hline $\mathrm{b}$ & $\mathrm{eV}$ & $-1.906+0.153 \mathrm{y}$ \\
\hline
\end{tabular}

Table 2 Calculated and measured ground state transition of $\mathrm{InGaAs} / \mathrm{InP}$ square quantum well

\begin{tabular}{|l|l|l|l|l|l|}
\hline $\begin{array}{l}\text { Well width } \\
(\mathrm{nm})\end{array}$ & $\begin{array}{l}\text { Experimental } \\
\text { value }(\mathrm{eV})\end{array}$ & $\begin{array}{l}\text { Calculated } \\
\text { value }(\mathrm{eV})\end{array}$ & $\begin{array}{l}\text { Error } \\
(\mathrm{meV})\end{array}$ & $\begin{array}{l}-6 \mathrm{meV} \text { for } \\
\text { exciton }\end{array}$ & $\begin{array}{l}-15 \mathrm{meV} \\
\text { for PL }\end{array}$ \\
\hline 5 & 1.2152 & 1.3751 & 159.9 & 153.9 & 138.9 \\
\hline 10 & 1.088 & 1.2719 & 183.9 & 117.9 & 162.9 \\
\hline 15 & 1.1235 & 1.1907 & 67.2 & 61.2 & 46.2 \\
\hline 20 & 0.9979 & 1.1272 & 129.3 & 123.3 & 108.3 \\
\hline
\end{tabular}

Table 3 The experimental data fitted $D_{b}, D_{w}$ and $k$ using the pseudo time model

\begin{tabular}{|l|l|l|l|}
\hline Time (hours) & $\mathrm{D}_{\mathrm{b}}\left(\mathrm{cm}^{2} / \mathrm{s}\right)$ & $\mathrm{D}_{\mathrm{w}}\left(\mathrm{cm}^{2} / \mathrm{s}\right)$ & $\mathrm{k}$ \\
\hline 2 & $2.3 \times 10^{-19}$ & $1.4 \times 10^{-11}$ & 25 \\
\hline 6 & $1.0 \times 10^{-20}$ & $1.0 \div 10^{-1 /}$ & 25 \\
\hline 8 & $2.2 \times 10^{-20}$ & $2.7 \times 10^{-1 /}$ & 20 \\
\hline
\end{tabular}




\section{Acknowledgment}

The authors acknowledge the financial support of the UGC-RGC Earmarked research Grant of Hong Kong.

\section{Reference}

1 R. J. Deri, E. Kapon, R. Bhat, M. Seto and K. Kash: Apl. Phys. Lett. 54, 1737 (1989)

2 I. Bar-Joesph, C. Klingshirn, D. A. B. Miller, D. S. Chemla, U. Koren and B. I. Miller: Appl."Phys. Lett. 50, 1010 (1987)

3 U. Koren. B. I. Miller, T. L. Koch, G. Eisenstein, R. S. Tucker, I. Bar-Joseph and D. S. Chemla: Appl. Phys. Lett. 51, 1132 (1987)

4 E. Herbert Li, W. C. Shiu, J. Micallef, Issac Ng, Jpn. J. Appl. Phys., 34, 1778-1783, (1995).

5 B. L. Weiss, E. Herbert Li, J. Micallef, J. Appl. Phys, 73, 11, (1993).

6 - G. C. Osbourn, J. Appl. Pgys. 53, 1586 (1982)

7 K. Nakashima, Y. Kawagtchi, Y. Kawamura, Y. Imamura. and h. asahi, appl. Phys. Lett. $52,1383(1988)$

8 T. Fujii, M. Sugawara, S. Yamazaki and K. Nakajima, J. Crys. G. 105, 348-352 (1990)

9 T. P. Pearsall, es,. GaInAsp Alloy Semicinductors (wiley, New York, 1982), p. 295.

10 S. A. Schwarz, P. Mei, T. Venkatesen, R. Bhat, D. N. Hwang, C. L. Schwarz, N. Koza, L. Nazar and B. J. Skromme, Appl. Phys. Lett. 53, 1051 (1988)

11 G. C. Osbourn, J. Appl. Phys. 53, 1586 (1982).

12 R. People, Appl. Phys. Lett. 50, 1604 (1987).

13 D. J. BenDaniel and C. B. Duke, Phys. Rev. 152, 682 (1966)

14 G. Bastard, J. B. Brum, and R. Ferreira, in Solid State Physics- Advances in Research and Applications, edited by H. Ehrenreich and D. Turnbull (Academic, New York, 1991), Vol. 44, p.232.

15 K. Mukai, M. Sugawara, and S. Yamazaki, Physical Review B, 50(4), 2273-2282, (1994).

16 M. B. Panish, H. Temkin, R. A. Hamm, and S. N. G. Chu, Appl. Phys. Lett. 49, 164 (1986)

17 R. Sauer, T. D. Harris, and W.T. Tsang, Phys. Rev. B 34, 9023 (1986)

18 David Gershoni and Henry Temkin, Journal of Lumin. 44, 381 (1989)

19 W. T. Tsang and E. F. Schubert, Appl. Phys. Lett. 49, 220 (1986)

20 Madelung, O. DST Semiconductors: Group IV Elements and III-V Compounds, Springererlag, Berlin, (1991).

21 Lawaetz, P. Physical Review B, 4(10), 3460-3467, (1971)

22 Qteish, A. and Needs, R.J. Physical Review B, 45(3), 1317-1326, (1992)

23 F. H. Julien, M. A. Bradley, E. V. K. Rac, M. Razeghi, and L, Gold-stein, Opt. Quantum Electron. 23, S847 (1991). 